\title{
Superhero Genes in Axolotl Limb Regeneration: Could Deadpool be Real?
}

\section{Atakan Z Namli* and Zeynep Gençyiğit}

Department of Molecular Biology and Genetics, Faculty of Arts and Science, Inonu University, Turkey

*Corresponding Author: Atakan Z Namli, Department of Molecular Biology and Genetics, Faculty of Arts and Science, Inonu University, Turkey.
Received: September 25, 2020

Published: October 30, 2020

(C) All rights are reserved by Atakan $\mathbf{Z}$

Namli and Zeynep Gençyiğit.

\section{Abstract}

Axolotls (Ambystoma Mexicanum) are vertebrates that have the ability to limb regeneration their body parts compared to most of other living populations. How this regeneration process works, how its mechanism takes place, and how blastemas are formed during differentiation are still being studied by researchers. Therewithal, it is known that many genes have significant effects in this regeneration process. Tgf- $\beta 1$, Fgf-8, Pax3 and Pax7, Lin28, Msx-2, HGF, Hoxb13 and Hoxc10 are some of these genes. It is also observed that some genes involved in the regeneration process in the human liver are common with some genes involved in limb regeneration in axolotls. Understanding this limb regeneration that occurs in axolotls is thought to have an inspiring importance in the treatment of limb amputation or loss as a result of various traumas in humans, especially in tissue engineering and regenerative medicine.

Keywords: Regeneration; Ambystoma Mexicanum; Liver Regeneration; Amputation; Proliferation; Dedifferentiation; Cell Growth; Blastema; Epithelium; Epidermal; Mesenchymal; Gene Expression; Progenitor

\section{Introduction}

Human being is an extremely complex and extraordinarily working mechanism. With its complex structure, gene sequences, DNA and incredibly functioning cells, it is like a multi-functional factory with trillions of workers. The human body's capacity to regenerate itself repeats itself in a mind-bogglingly miraculous way. Heart muscles that continue to work tirelessly from the embryo period to death, nails that grow an average of $3 \mathrm{~mm}$ every month, colon epithelial cells that renew themselves every

5 days, red blood cells with an average life of 120 days and completely white blood cells involved in renewed immunity. In addition, this magnificent structure is accompanied by this magnificent structure in our body parts that are restored to their original state as a result of the perfect self-repair of some organs and tissues that are damaged in an unnatural way (by damages from the external environment, cuts, blows, etc.). But as a human species, this selfrenewal and repair of damaged or ruptured organs is limited. As humans, we are constantly working to gain the ability to heal and use our dysfunctional organs or tissues, and we do not hesitate to use the possibilities offered by technology at this point. But there is a creature among vertebrates that is extremely functional in regenerating and repairing broken organs, which has the ability to regenerate body organs, including the degraded spinal cord and arm, especially the tail: Axolotl.

During the Devonian, about 400 million years ago, the first tetrapods began exploring the land. During this period, they spread to several species, including two types of amphibians: frogs (anuran tailless) and salamanders (urodeles - showing a tail). While all amphibians showed regenerative properties, some of these showed better regenerative effects than others [1,2]. The indiscriminate apparition of pervasive regeneration capacities in the animal kingdom points to a phylogenetically widespread regeneration potential masked in most species [3-7]. Among tetrapods, salamanders, such as newts and axolotls, exhibit the largest regenerative reper- 
toire [8]. A salamander can rebuild entire limbs, tails, jaws, heart muscle, ocular tissues and repair central nervous system tissues including brain structures. However, it is important to note that there are large differences even between salamanders [9].

Axolotl limb regeneration is thought by many to be divided into two main stages [10-12]. The first stage is called the preparatory stage and begins immediately after amputation with the formation of a wound epithelium (WE) on the amputation plane. Cellular differentiation and migration also occurs at this stage, which will eventually lead to the formation of a regeneration blastema, also take place in this phase explosion. In the second phase of limb regeneration, called the regeneration phase, the blastema cells stop reproducing and begin to differentiate again to regenerate the lost part $[12,13]$. During the preparation phase, there is extensive remodeling of the extracellular matrix (ECM) through the action of tissue inhibitors of matrix metalloproteinases (MMP) and metalloproteinases, as in mammalian wound healing, following WE formation [14,15]. MMP inhibitors such as GM6001 have been shown to inhibit limb regeneration and cause the appearance of a scar-like skin layer with collagen layer on the stumps of amputated limbs [14].

What are the obstacles to cell renewal in humans and what allows this renewal event in other organisms? Among vertebrates, salamanders, such as newts, have been instrumental in many of the basic studies in regenerative biology [16]. They are distinguished by their exceptional regenerative abilities and are known for their ability to regenerate full limbs upon amputation [17]. The limb provides a particularly good system for working the regeneration process because it needs to be simply accessible for experimental manipulation and allows for non-invasive monitoring of regeneration.

The reason why this creature attracts such attention of scientists and is the subject of their research is that it can restore body parts as a 'stem cell' element, as stated above. Axolotls are neotenic creatures. This means they cannot undergo metamorphosis, they have gills and adults live in water. They are vertebrates and are found in the salamander group of amphibians.

There are studies about how axolotls do this regeneration, which genes or hormones are effective. For example, according to a study by Mathieu Lévesque., et al. (2007) [18], they found that transforming growth factor-beta family (TGF- $\beta$ ) is effective in limb regeneration in axolotls. At the same time, in the study conducted by Man-Jong Han., et al. (2001) [19], there are studies on Fgf-8 and Carlson MRJ., et al. (2001) [20] that expression of the Hox genes family plays a role in axolotl organ regeneration and development. We will discuss these studies in detail later.

\section{Background}

Limb regeneration in salamanders involves a complex process involving several morphological stages. but we can create subject with three main status. Following amputation, the stump tissue responds to the injury with a rapid wound improvement phase in which epithelial cells cover the open wound tissue and form the wound epidermis. These ensure significant signals to induce blastema formation, a heterogeneous mass of mesenchymal cells formed at the wound region caused from stump tissues. These mesenchymal cells will then multiply, re-differentiate and continue to form new attachment through morphogenesis [21,22]. This represents a classic feature of epimorphic regeneration as the regenerative effect is associated with a blastema [23].

The wound epidermis, which begins to unite shortly after amputation, is created by the displacement of epidermal basal cells. While the migrating cells do not multiply [24], a group that divides the epidermal cell placed close to the wound site provides a permanent flow of migrating cells [25-27]. As the blastema cells pile up, thickening of the epidermis can be allowed, which will then allow the formation of the apical epidermal cover. The AEC is a structure similar to the apical ectodermal ridge (AER) of amniotic growing limbs that functions as a distal signaling center that warn blastema proliferation [28]. The prominence of the wound epidermis is well documented in early studies showing that removal or mechanical degradation of this structure can estop regeneration [29].

\section{Genome size}

The mexicanum HoxA cluster has significantly increased repeat content and is 3.5 times larger, mostly due to a $170 \mathrm{~kb}$ enlargement between HoxA3 and HoxA4. In particular, highly conserved nonexonic elements assumed to overlap with cis-regulatory elements are not sprinkle in this $170-\mathrm{kb}$ area, but stay close to HoxA3 and HoxA4. The axolotl has a typical HoxA gene structure with two coding exons discrete by an intron. In particular, in unlike to the general expansion of intron sizes, the intron sizes at the axolotl HoxA locus are also very similar to other vertebrates barring AmHoxA3, which is the longest of the HoxA genes in other tetrapods. distin- 
guished HoxC and HoxD genes examined in red spotted salamender showed similar characteristics [30].

The introns of the non-developmental genes in the axolotl show an average size extension of 13 to 25 times check against to the human, mouse, and frog, while the expansion of the introns of the developmental genes is significantly less. Unlike human, mouse, and frog, the introns of developmental genes in axolotl are shorter than introns of non-developmental genes. Moreover, axolotl multi-exon genes containing only short introns exhibit unenriched, developmental modeling- related gene ontology enrichments in multiple exon genes with larger introns [31]. Out of these effects especially, those mainly split between retrotransposons (class I) and DNA transposons (class II) [32] have been shown to include $47 \%$ of the entire genome in some species [33]. Additionally, this same work discovered that the long terminal repeat (LTR) retrotransposon is a highly symbolized part, explaining approximately one-third of the genome. The presence of these symbolized excessively elements supply relevant aspirant to be investigated in the terms of regeneration. Logical with these hypothesis, another family of retrotransposons, the non-LTR long sprinkled nucleotide element-1 (LINE-1), has a short time since played a role in axolotl limb regeneration [34]. Work discovered that LINE-1, which is habitually active in germ cells, is highly regulated during limb regeneration and occur a germ-like state that may play a role in inducing cell dedifferentiation. An exception to this rule is the Hox gene cluster: considering the general rise in intron length, the sizes of the introns at the axolotl HoxA region are very look like to those in other vertebrates [35].

\section{The Importance of TGF- $\beta 1$ in Limb Regeneration}

The relationship between the healing process of any damaged tissue or the self-renewal process of a severed limb has always been a matter of curiosity. Many studies have discussed the similarities between regeneration and wound healing [10,36]. However, it is possible to observe that regeneration stages in axolotls generally take place in a two-stage $[10,37,38]$ phase: In the first stage, the formation of wound epithelium and covering of the open tissue and blastema regeneration by cell differentiation [39]. In the second stage, the cells are re-differentiated enough to regrowth and limb development $[40,41]$. However, there are similarities between limb regeneration in axolotls and wound healing in mammals [42]. Tgf- $\beta 1$ gene (supplementary 1 gene coding), which is a very important regulator, is the gene that produces the protein named Tgf- $\beta 1$, which performs many functions such as cell growth, differentiation, proliferation and apoptosis $[43,44]$. Although there are many members of the Tgf- $\beta$ family responsible for growth and differentiation, Tgf- $\beta 1$ is the most studied during the wound healing process in mammals $[45,46]$. Many previous studies have also shown that the inhibitor named SB-431542 inhibits Tgf- $\beta$ and Tgf- $\beta 1[47,48]$ at the same time Levesque., et al. in the study carried out by, they concluded that the inhibitor named SB-431542 terminates limb regeneration in axolotls and actually Tgf- $\beta$ is absolutely necessary for regeneration [49]. According to the study, the first Tgf- $\beta 1$ expression in the amputated axolotl limb is observed at a rate of almost no to no at 6 th hour after amputation. At 48 hours after amputation, severe Tgf- $\beta 1$ is observed. To see if Tgf- $\beta$ signaling is essential for axolotl regeneration, it is treated with SB-431542. According to the findings, limb regeneration is completely blocked after SB-431542 [50].

\section{The role of Fgf-8 in limb regeneration}

Fgf-8(Supp.2 gen cod.) is a protein encoded by the HBGF (heparin-binding growth factor) family of the FGF (fibroblast growth factor) gene. The Fgf gene is involved in many biological activities, including embryonic development, growth, tissue repair and tumor growth [51]. During the development of the limb in axolotls, Fgf-8 begins to be expressed in the forefoot area and in the limb regions that have just begun to develop, and it is observed that the gap where it is produced most is formed in the mesenchymal tissues before finger formation [19]. Fgf-8 expression was found to be in the mesenchymal region rather than the axolotls epidermis region [19]. Analyzed the expression of Fgf-8 in embryonic development and organ regeneration by cloning the homologue of the Fgf-8 protein of the Mexican axolotl according to their study. The axolotl Fgf-8 has two putativ N (asn) -linked glycosylation sites [19]. Expression of Fgf- 8 was observed in the first phase of tail formation, in the gills, in the isthmus, and in the early phase of facial formation (in which very intense expression was observed in these regions) [19]. Compared to other vertebrates, the expression pattern of Fgf- 8 in axolotls is quite specific, with other vertebrates Fgf- 8 is initially expressed in the limb regions that first formed in the epidermis [19]. However, when axolotl larvae were amputated from the elbow regions, no expression of Fgf- 8 was observed until dedifferentiation started [19]. Later, Fgf-8 expression was detected in a very low amount in the early bud stage of blastema regeneration [19]. Axolotls expressed the highest amount of Fgf-8 in the medium bud stage during blastema formation [19]. 
Msx-2 expression in axolotls

Msx-2(Supp.3 gen cod.) is a protein encoded by MSX2 genes [52]. Msx-2 expression generally causes cell proliferation, cell differentiation and cell migration during embryogenesis [53]. The msx-2 cDNA blastema regeneration in axolotls was described by Gardiner., et al. (1995) in their library. M.R.J. Carlson., et al. in their study, the axolotl clearly forms the 5 'sequence clones into the homeobox they isolated, homologous to Msx-2 and Msx-2 expression was detected as a single transcript of approximately $3.3 \mathrm{~kb}$ of total RNA isolated from axolotl tissues [54]. In the study, quite low Msx2 expression was detected in mature limbs, while the most intense interval was observed as the medium bud phase. However, expression was also found in the epidermal and mesenchymal components of the blastema. Cells generally express Msx-2 when signals are released from BMPs molecules [55]. Because BMPs are found in the developing skin (Chuong., et al. 1996; Stelnicki., et al. 1998), trauma alone is not sufficient for Msx-2 compression and expression is not initiated as the injured tissue is immediately covered with a thick skin layer after amputation [56].

Expression only occurs as a result of any contact of the wound epidermis with injured mesenchymal tissues [56].

\section{Hoxb13 and Hoxc10 expression in axolotls}

Hox genes are genes that determine which body part goes where in the embryo along the head-tail axis in animals. In most animals, the organizations of the Hox genes on the chromosome are the same in the order of their expression along the anteroposterior axis of the developing animal [57]. To understand their role in body development and regeneration in axolotls, Carlson MRJ., et al. (2001) conducted a study comparing the expression of Hoxb13 (Supp.4 gen cod.) and Hoxc10 genes (Supp.5 gen cod.) [58]. According to this study, unlike other developed vertebrates, Hoxb13 was detected not only in the developing tail tip, but also in the distal mesenchyme of the developing hind limbs and in small amounts in the developing forelimbs. Hoxc10 gene is expressed in both development and regeneration.

While both of the Hoxb13 and Hoxc10 genes were not found in the mature tail, hoxb13 was expressed in the tail blastema mesenchyma, while hoxc10 was found at a very high level in the regenerated tail blastema [58]. Hoxb13 expression was first observed on the 3rd day after amputation, while Hoxc10 expression was first observed during differentiation in stage 2 tail blasting (Iten and
Bryant, 1976) [59]. The most intense interval of expression in the two genes was determined as 5-7 days after amputation [58].

\section{Pax3/Pax7 gene}

These genes are included of the family of paired box (PAX) transcription factors. Despite the presence of Pax3(Supp.6 gen cod.) and Pax7 (Supp.7 gen cod.) paralogs in all other known vertebrate lineages, we were able to identify Pax7 in the axolotl genome assembly, but not Pax3 [31]. Axolotl Pax7 has parallel task to Pax3. To functionally evaluate the result of the lack of Pax3 in the axolotl, They used TALEN and CRISPR by way of gene editing to mutate Pax7 [60]. In another vertebrates, Pax3 and Pax7 play a significant part in muscle, neural tube, and neural crest- derived tissue development [61]. Forfeit of function works using TALEN and CRISPR/ Cas9 genome editing in axolotls show that Pax7 paralogs play the role of Pax3 in other vertebrates, as Pax7 axolotl mutants have large developmental abnormalities and deficiency limb muscle [35]. In contrast, the Pleurodeles genome contains both Pax3 and Pax7. The loss of Pax3 in Pleurodeles, as in other vertebrates, causes serious risks, including skeletal muscle agenesis. Although Pax7 is definitely, necessary for accomplished skeletal muscle regeneration in mammals, the function in Pleurodeles does not reason any substantial regeneration phenotype. This status might show that, in the lack of Pax7, skeletal muscle regeneration is fueled by dedifferentiation of myofibres in Pleurodeles [62].

Skeletal muscle regeneration is organized by families of transcription factors that are also necessary for skeletal muscle nascency in the embryo. Pax3, a paired box transcription factor, is expressed in the paraxial mesoderm of the nascent somites and then in the dorsal compartment of the somites in the dermomyotome, after which it gives rise to myogenic progenitor cells. Pax3 and its similar gene Pax7, which is later expressed in all muscle progenitor cells, is located upstream of the MYOD family of basic-helix-loophelix transcription factors that play an important role in muscle formation. The MYOD family is specific to skeletal muscle, Pax3 and Pax7 are also expressed in some cells of the central nervous system, and Pax3 plays an key role in the growth of neural crest cells [63]. In the context of skeletal muscle, the population of Pax3/ Pax7-positive progenitors is necessary for myogenic develop during development and is also thought the weld of adult Muscle stem cells (MuSCs) [64]. in these days research has revealed that core transcription factors expressed in adult stem cells of a number of different tissues can directly induce and sustain a particular cell 
line when consolidated into pluripotent stem cells (PSCs) and another cell types [65]. A standard example of such tissue-specific transformation is provided by the MYOD family, which activates endogenous MyoD family genes when expressed in extra-muscular cells, leading to the formation of the gene regulatory network required for following skeletal muscle formation [66].

\section{Lin28 gene}

Lin28A (Supp.8 gen cod.) and Lin28B (Supp.9 gen cod.) are highly protected RNA binding and proteins organized by microRNA. Generally, they selectively block the expression of members of the let-7 microRNA family that act as tumor suppressors by inhibiting the expression of key regulators of oncogenes and mitogenic pathways, including RAS, MYC and HMGA2 [67]. Lin28A recruits TUTase to inhibit let-7 precursors to block Dicer processing in the cell cytoplasm, while Lin28B suppresses let-7 maturation via a TUTase independent mechanism [68]. According to the research have shown that both Lin28A and Lin28B show up-regulated expression in human malignancies and function as oncogenes by promoting transformation and tumor progression [69,70]. LIN28 is heard for its role in induced pluripotent stem cells (iPSCs). Together with NANOG, OCT4 (POU5F1) and SOX2, it can reprogram somatic cells into pluripotent stem cells [71].

OCT4 and SOX2 have been shown to be essential components for this induction commit, while LIN28 and NANOG are unnecessary factors. LIN28 and NANOG each rise reprogramming performance while triggering differentiation NANOG to a large extent than LIN28 [71]. To assistance this proposed role, LIN28 has been represent, to promote increased reprogramming by inducing large cell division rates and an increased number of cell divisions [72].

In the tissue regeneration research, LIN28 overexpression results in increased finger renovation, epidermal hair regrowth and pinnal tissue regrowth in the mouse. The, achievement of tissue regeneration may depend on LIN28 mediated control of stem cell proliferation and transit amplifier populations [73]. LIN28 promotes the dilation of these cell types leading to an increase in tissue size and regrowth of the wounded area.

\section{HGF gene}

Acute liver injury is a protein factor found in animal plasma. HGF (Supp.10 gen cod.) can stimulate the liver cell to synthesize DNA and also plays an key role in the liver regeneration process. Its active ingredient is called HPTA (Hepatopoietin). HGF is mainly found in Brunner glands of pancreatic acinar cells, neuron, thyroid cells, Salivary pancreatic duct cells and duodenal pancreatic duct cells. Through the bloodstream, HGF take part in the regulation of liver cell growth. Other method to examine HGF distribution in vivo is HGF-mRNA detection. The result indicate, that HGF-mRNA is available in rat lung, liver and brains. In humans, HGF is also present in placenta and embryonic lung fibroblasts [74]. The liver has several important functions in body metabolism and is capable of actively regenerating during partial hepatectomy or hepatitis. Cross-circulation experiments in parabiotic rodents show that liver regeneration is initiated by a blood-borne factor, so many biologists say it is the causative agent that initiates liver regeneration after $70 \%$ hepatectomy $[75,76]$. tried to identify a ghost factor. found that adult rat hepatocytes in primary culture retained a large number of liver-specific functions and responded to a variety of hormones, and this fact led to the identification of hepatotrophic factor (s), as yet unknown at the time of establishment.

A number of in vitro studies have revealed that HGF has regenerative effects on epithelium in kidney, lung, and other tissues [7780]. Indeed, plasma HGF levels increase in patients during organ injuries [81]. The paracrine or endocrine pathway, which is mainly involved in tissue repair, depends on the degree or type of injury. Regardless of the route, HGF is secreted as a pro-HGF and then converted into active form only at the injury sites by HGF activators $[78,81]$.

It acts as a main mitogenic stimulator directing hepatocytes towards proliferation. We thought that ablation of HGF in adult mice would have a negative effect on the regenerative ability of hepatocytes. Deletion of the HGF gene was accomplished by inducing systemic recombination in mice lacking exon 5 of HGF and carrying the Mx1-cre or Cre-ERT transgene. Analysis of liver genomic DNA obtained from animals 10 days after treatment showed that the majority (70-80\%) of alleles underwent induced genetic recombination. Surprisingly, however, analysis by RT-PCR showed that both non-recombined and recombined forms of HGF mRNA continued to exist after treatment. Hepatocyte growth factor (HGF) has been shown to be indispensable for liver regeneration because it serves as a main mitogenic stimulus driving hepatocytes toward proliferation [82].

Apoptotic events are one of the key pathogenic causes for the apparition of organ damage. These effects of HGF take care of epithelium, neurons and cardiomyocytes in the course of organ dis- 
eases. HGF is considered a hepato-regenerative factor in the course of liver damage $[83,84]$. HGF is considered a hepato-regenerative factor in the course of liver damage. Therefore, it is important to elucidate the mechanism by which host cells occur and deliver HGF to damaged region as a response proportional to the type and size of liver damage. Proinflammatory cytokines can trigger HGF production in intrahepatic tissues (i.e. as a paracrine ring) and extrahepatic organs (i.e. as an endocrine system). Additionally, humoral mediators such as neurotransmitters, hormones, and autocoids can be play a role in the endocrine mechanism of HGF production $[85,86]$.

\section{Effects of progenitor cells on regeneration}

There are many types of progenitor cells in the human body. Every progenitor cell can differentiate only into cells belonging to the same tissue or organ. Some progenitor cells have one recent target cell to which they differentiate, while an others have the power to terminate in more than one cell type [87]. In humans, chronic liver disease such as acute liver injury or late stage cirrhosis causes the active of progenitor cells [88]. Like oval cells, human progenitor cells or LSPCs are include in Hering ducts [89,90]. A research conducted with biopsies from patients with massive hepatic necrosis occurring after departmental liver transplantation prove the important role of LSPCs in parenchymal regrowth after acute liver failure [91]. The important role of LSPCs in liver regeneration has received more experimental and clinical assistance. For example, positive results after acetaminophen-induced liver injury appear to be directly related to serum alpha-fetoprotein level [92].

\section{Discussion and Conclusion}

To be alive means to be in a state of constant change. Regeneration. Our body is in a state of constant renewal and change from its functioning at the atomic level to the cellular and finally at the organ level. Thanks to the perfect mechanism of our genes some of our parts continue to grow in perfect harmony for a lifetime. There is a certain limit to the growth of our body parts such as growth of our height, organs and teeth. However, although some of our body parts, such as hair and nails, they have the potential to regrow for a lifetime. What if our severed arm or cutted leg could grow back to its former state, just like our hair that can grow back when we cut it?

Axolotls are one of the rare creatures that can achieve this perfect regeneration. In fact, there is an organ in our body that can perform this function as close to this regeneration in axolotls: The liver. Many genes and proteins involved in limb regeneration in axolotls jointly take part in liver regeneration. Some of the genes and proteins we mentioned earlier, such as Tgf- $\beta 1$, HGF, FGF, are actively involved in liver regeneration [93]. While Tgf- $\beta 1$ has important functions such as cell growth and differentiation, it has been determined that it has an essential role in regeneration when limb regeneration in axolotls did not occur after it was inhibited with the inhibitor called SB-431542 as we mentioned earlier. If we look at it from a different perspective, this result might raise the question: If Tgf- $\beta 1$ (which is also involved in liver regeneration), which is responsible for cell proliferation, and can be inhibited by using SB431542, can we achieve the same effect on cancer cells that grow uncontrollably using the same inhibitor? As we mentioned earlier, LSPCs have been found to have the ability to differentiate into hepatocytes and bile duct cells. Accordingly, it is thought that after transplantation, some of the LSPCs will differentiate into hepatocytes and bile duct cells upon nesting in the liver of the recipient. Other than that, studies in the field of regenerative medicine are carried out on damaged tissue regeneration, especially the treatment of organ failure. The investigations aim that organs and amputated limbs other than the liver can regenerate themselves as a result of these studies similar to axolotls [94-97].

As a result, we believe that this enormous regeneration process carried out by these living beings called axolotls will provide an alternative approach to the current study process by providing another perspective to the treatment and research studies in many fields, especially in cancer, tissue engineering and stem cell studies.

Supplementary Information

- https://www.genecards.org/cgi-bin / carddisp. pl?gene=TGFB1\&keywords=TGF-,1

- https://www.genecards.org/cgi-bin / carddisp. pl?gene=FGF8\&keywords=Fgf-8

- https://www.genecards.org/cgi-bin / carddisp. pl?gene=MSX2\&keywords=Msx-2

- https://www.genecards.org/cgi-bin / carddisp. pl?gene=HOXB13\&keywords=hoxb13

- https://www.genecards.org/cgi-bin / carddisp. pl?gene=HOXC10\&keywords=hoxc10

- https://www.genecards.org/cgi-bin/carddisp. pl?gene=PAX3\&keywords $=$ pax3

- https://www.genecards.org/cgi-bin/carddisp. pl?gene=PAX7\&keywords $=$ pax 3 
- https://www.genecards.org/cgi-bin / carddisp. pl?gene=LIN28A\&keywords=Lin28

- https://www.genecards.org/cgi-bin / carddisp. pl?gene=LIN28B\&keywords=Lin28

- https://www.genecards.org/cgi-bin/carddisp. pl?gene=HGF\&keywords=hgf

\section{Bibliography}

1. Tanaka E M. "Regeneration: if they can do it, why can't we?" Cell 113 (2003): 559-562.

2. Yun M H. "Changes in regenerative capacity through lifespan". International Journal of Molecular Sciences 16 (2015): 2539225432.

3. Tanaka E M. "The molecular and cellular choreography of appendage regeneration". Cell 165 (2016): 1598-1608.

4. Gemberling M., et al. "The zebrafish as a model for complex tissue regeneration". Trends Genetics 29 (2013): 611-620.

5. Galliot B and Chera S. "The Hydra model: disclosing an apoptosis-driven generator of Wnt-based regeneration". Trends in Cell Biology 20 (2010): 514-523.

6. Roberts-Galbraith R H and Newmark P A. "On the organ trail: insights into organ regeneration in the planarian". Current Opinion in Genetics and Development 32 (2015): 37-46.

7. Brockes JP. “Amphibian limb regeneration: rebuilding a complex structure". Science 276 (1997): 81-87.

8. Simon A and Tanaka E M. "Limb regeneration". Wiley interdisciplinary 2 (2013): 291-300.

9. Sergej Nowoshilow., et al. "The axolotl genome and the evolution of key tissue formation regulators". Nature (2018).

10. Roy S and Levesque M. "Limb regeneration in axolotl: is it super healing?" Scientific World Journal 6 (2006): 12-25.

11. Gardiner DM., et al. "Regulation of HoxA expression in developing and regenerating axolotl limbs". Development 121 (1995): 1731-1740.

12. Gardiner DM., et al. "Towards a functional analysis of limb regeneration". Seminars in Cell and Developmental Biology 10 (1999): 385-393.

13. Wallace H. "Vertebrate limb regeneration". Chichester Eng.; New York: Wiley (1981): 276.
14. Vinarsky V., et al. "Normal newt limb regeneration requires matrix metalloproteinase function". Development Biology 279 (2005): 86-98.

15. Madlener M. "Differential expression of matrix metalloproteinases and their physiological inhibitors in acute murine skin wounds". Archives of Dermatological Research 290 (1998): S24-29.

16. Jeremy Brockes Anoop Kumar. "Comparative Aspects of Animal Regeneration" (2008).

17. David L. "Stocum: Mechanisms of urodele limb regeneration" (2017).

18. Le'vesque M., et al. "Transforming Growth Factor: b Signaling Is Essential for Limb Regeneration in Axolotls". PLOS ONE 2.11 (2007): e1227.

19. Han MJ., et al. "Expression Patterns of Fgf-8 During Development And Limb Regeneration of the Axolotl". Developmental Dynamics 220 (2001): 40-48.

20. Carlson MRJ., et al. "Expression of Hoxb13 and Hoxc10 in Developing and Regenerating Axolotl Limbs and Tails". Developmental Biology 229 (2001): 396-340.

21. Brockes JP and Kumar A. "Plasticity and reprogramming of differentiated cells in amphibian regeneration". Nature Reviews Molecular Cell Biology 3 (2002): 566-574.

22. Iten LE, Bryant SV. "Forelimb regeneration from different levels of amputation in the newt, Notophthalmus viridescens: length, rate, and stages". Wilhelm Roux Arch Entwickl Mech Org 173.4 (1973): 263-282.

23. Carlson B M. "Principles of Regenerative Biology". Academic Press (2007).

24. Hay ED and Fischman D A. "Origin of the blastema in regenerating limbs of the newt Triturus viridescens. Anautoradiographic study using tritiated thymidine to follow cell proliferation and migration". Developmental Biology (1961).

25. Repesh L A and Oberpriller J C. "Scanning electron microscopy of epidermal cell migration in wound healing during limb regeneration in the adult newt, Notophthalmus viridescens". American Journal of Anatomy (1978).

26. Repesh L A and Oberpriller J C. "Ultrastructural studies on migrating epidermal cells during the wound healing stage of regeneration in the adult newt, Notophthalmus viridescens". American Journal of Anatomy (1980). 
27. Lash JW. "Studies on wound closure in urodeles". Journal of Experimental Zoology (1955).

28. Stocum D L. "Mechanisms of urodele limb regeneration" (2017).

29. Goss R J. "Principles of Regeneration". New York: Academic Press (1969).

30. Voss S R., et al. "Salamander Hox clusters contain repetitive DNA and expandednon-coding regions: a typical Hox structure for non-mammalian tetrapodvertebrates". Human Genomics 7 (2013).

31. Sergej Nowoshilow., et al. "The axolotl genome and the evolution of key tissue formation regulators". Nature 554.7690 (2018).

32. Wicker T., et al. "A unified classification system for eukaryotic transposable elements". Nature Reviews Genetics 8 (2007): 973-982.

33. Sun C., et al. "LTR retrotransposons contribute to genomic gigantism in plethodontid salamanders". Genome Biology and Evolution (2012).

34. Smith JJ., et al. "Genic regions of a large salamander genome contain long introns and novel genes". BMC Genomics 10 (2009): 19.

35. Gerber T., et al. "Single-cell analysis uncovers convergence of cell identities during axolotl limb regeneration". Science (2018).

36. Goss RJ. "Prospects of regeneration in man". Clinical Orthopaedics and Related Research (1980): 270-282.

37. Gardiner DM., et al. "Regulation of HoxA expression in developing and regenerating axolotl limbs". Development 121 (1995): 1731-1741.

38. Gardiner DM., et al. "Towards a functional analysis of limb regeneration". Seminars in Cell and Developmental Biology 10 (1999): 385-393.

39. Le'vesque M., et al. "Transforming Growth Factor: b Signaling Is Essential for Limb Regeneration in Axolotls". PLOS ONE 2.11 (2007): e1227.

40. Wallace H. "Vertebrate limb regeneration". Chichester Eng.; New York: Wiley 12 (1981): 276.

41. Gardiner DM., et al. "Towards a functional analysis of limb regeneration". Seminars in Cell and Developmental Biology 10 (1999): 385-393.
42. Le'vesque M., et al. "Transforming Growth Factor: b Signaling Is Essential for Limb Regeneration in Axolotls". PLoS ONE 2.11 (2007): e1227.

43. Ghadami M., et al. "Genetic mapping of the Camurati-Engelmann disease locus to chromosome 19q13.1-q13.3". American Journal of Human Genetics 66.1 (2000): 143-147.

44. Vaughn SP., et al. "Confirmation of the mapping of the Camurati-Englemann locus to $19 \mathrm{q} 13.2$ and refinement to a 3.2-cM region". Genomics 66.1 (2000): 119-121.

45. Massague J., et al. "TGFbeta signaling in growth control, cancer, and heritable disorders". Cell 103 (2000): 295-309.

46. O'Kane S., et al. "Transforming growth factor beta s and wound healing". The International Journal of Biochemistry and Cell Biology 29 (1997): 63-78.

47. Callahan JF., et al. "Identification of novel inhibitors of the transforming growth factor beta1 (TGFbeta1) type 1 receptor (ALK5)". Journal of Medicinal Chemistry 45 (2002): 999-1001.

48. Inman GJ., et al. "SB-431542 is a potent and specific inhibitor of transforming growth factor-betasuperfamily type I activin receptor-like kinase (ALK) receptors ALK4, ALK5, and ALK7". Molecular Pharmacology 62 (2002): 65-74.

49. Le'vesque M., et al. "Transforming Growth Factor: b Signaling Is Essential for Limb Regeneration in Axolotls". PLoS ONE 2.11 (2007): e1227.

50. Le'vesque M., et al. "Transforming Growth Factor: b Signaling Is Essential for Limb Regeneration in Axolotls". PLOS ONE 2.11 (2007): e1227.

51. Hao Y., et al. "Roles of FGF8 subfamily in embryogenesis and oral-maxillogacial diseases (Review)". International Journal of Oncology 54 (2019): 797-806.

52. Takahashi C., et al. "Characterization of a human MSX-2 cDNA and its fragment isolated as a transformation suppressor gene against v-Ki-ras oncogene". Oncogene 12.10 (1996): 21372146 .

53. Liu H., et al. "microRNA-203 promotes proliferation, differentiation, and migration of osteoblasts by upregulation of Msh homeobox 2". Journal of Cellular Physiology 234.10 (2010): 17639-17648.

54. Carlson MRJ., et al. "Expression of Msx-2 During Development, Regeneration, and Wound Healing in Axolotl Limbs". Journal of Experimental Zoology 282 (1998): 715-723. 
55. Rifas L. "Gestational exposure to ethanol suppresses msx2 expression in developing mouse embryos". Proceedings of the National Academy of Sciences of the United States of America 94.14 (1997): 7549-7554.

56. Carlson MRJ., et al. "Expression of Msx-2 During Development, Regeneration, and Wound Healing in Axolotl Limbs". Journal of Experimental Zoology 282 (1998): 715-723.

57. Pearson JC., et al. "Modulating Hox gene functions during animal body patterning". Nature Reviews Genetics 6.12 (2010): 893-904.

58. Carlson MRJ., et al. "Expression of Hoxb13 and Hoxc10 in Developing and Regenerating Axolotl Limbs and Tails". Developmental Biology 229 (2001): 396-340.

59. Iten LE and Bryant SV." Stages of tail regeneration in the adult newt, Notophthalmus viridescens". Journal of Experimental Zoology 196 (1976b): 283-292.

60. Gaj T., et al. "3rd ZFN, TALEN, and CRISPR/Cas-based methods for genome engineering". Trends in Biotechnology.

61. Mansouri A., et al. "Pax genes and their roles in cell differentiation and development". Current Opinion in Cell Biology 8.6 (1996): 851-857.

62. Elewa A., et al. "Reading and editing the Pleurodeles waltl genome reveals novel features of tetrapod regeneration". Nature Communications 8 (2017).

63. Buckingham M and Relaix F. "PAX3 and PAX7 as upstream regulators of myogenesis". Seminars in Cell and Developmental Biology 44 (2015): 115-125.

64. Gros J., et al. "A common somitic origin for embryonic muscle progenitors and satellite cells". Nature 435 (2005): 954-958.

65. Morris SA. "Direct lineage reprogramming via pioneer factors; a detour through developmental gene regulatory networks". Development 143 (2016): 2696-2705.

66. Weintraub H., et al. "Activation of muscle-specific genes in pigment, nerve, fat, liver, and fibroblast cell lines by forced expression of MyoD". Proceedings of the National Academy of Sciences of the United States of America 86 (1989): 5434-5438.

67. Bussing I., et al. "Let-7 microRNAs in development, stem cells and cancer". Trends in Molecular Medicine 14.9 (2008): 400409.
68. Piskounova E., et al. "Lin28A and Lin28B inhibit let-7 microRNA biogenesis by distinct mechanisms". Cell 147.5 (2011): 1066-1079.

69. Hsu KF., et al. "Overexpression of the RNA-binding proteins Lin28B and IGF2BP3 (IMP3) is associated with chemoresistance and poor disease outcome in ovarian cancer". British Journal of Cancer 113.3 (2015): 414-424.

70. Tu HC., et al. "LIN28 cooperates with WNT signaling to drive invasive intestinal and colorectal adenocarcinoma in mice and humans". Genes Development 29.10 (2015): 1074-1086.

71. Yu J., et al. "Induced pluripotent stem cell lines derived from human somatic cells". Science 318 (2007): 1917-1920.

72. Hanna J., et al. "Direct cell reprogramming is a stochastic process amenable to acceleration". Nature 462 (2009): 595-601.

73. Shyh-Chang N., et al. "Lin28 enhances tissue repair by reprogramming cellular metabolism”. Cell 155 (2013): 778-792.

74. Nakamura T and Aoyama K. Biochemical and Biophysical Research Communications 91 (1979): 515-520.

75. Nakamura T., et al. "Precocious induction of glucokinase in primary cultures of postnatal rat hepatocytes". Biochemical and Biophysical Research Communications 91.2 (1979): 515-520.

76. Forte TM. "Primary hepatocytes in monolayer culture: a model for studies on lipoprotein metabolism”. Annual Review of Physiology 46 (1984): 403-415.

77. Nakamura T. "Structure and function of hepatocyte growth factor". Progress in Growth Factor Research 3 (1991): 67-85.

78. Matsumoto $\mathrm{K}$ and Nakamura T. "Hepatocyte growth factor: molecular structure, roles in liver regeneration, and other biological functions". Critical Review on Oncogenesis 3 (1992): 27-54.

79. Mizuno and Nakamura T. "Hepatocyte growth factor: a regenerative drug for acute hepatitis and liver cirrhosis". Regenerative Medicine 2 (2007): 161-170.

80. Mizuno S., et al. "HGF as a renotrophic and anti-fibrotic regulator in chronic renal disease". Frontiers in Bioscience 13 (2008): 7072-7086.

81. Funakoshi $\mathrm{H}$ and Nakamura T. "Hepatocyte growth factor: from diagnosis to clinical applications". Clinica Chimica Acta 327 (2003): 1-23. 
82. Kari Nejak-Bowen., et al. "Conditional genetic elimination of hepatocyte growth factor in mice compromises liver regeneration after partial hepatectomy". Plos One (2013).

83. Kosai K., et al. "Abrogation of Fas-induced fulminant hepatic failure in mice by hepatocyte growth factor". Biochemical and Biophysical Research Communications 244 (1998): 683-690.

84. Yamamoto K., et al. "Contribution of Bcl-2, but not Bcl-xL and Bax, to antiapoptotic actions of hepatocyte growth factor in hypoxia-conditioned human endothelial cells". Hypertension 37 (2001): 1341-1348.

85. Ekberg S., et al. "Growth hormone promotes early initiation of hepatocyte growth factor gene expression in the liver of hypophysectomized rats after partial hepatectomy". Journal of Endocrinology 135 (1992): 59-67.

86. Matsumoto K., et al. "Identification and characterization of "injurin". an inducer of expression of the gene for hepatocyte growth factor". Proceedings of the National Academy of Sciences of the United States of America 89 (1992): 3800-3804.

87. Nicole Gleichmann. "What are Progenitor Cells? Exploring Neural, Myeloid and Hematopoietic Progenitor Cells". technologynetworks.com

88. L Libbrecht and T Roskams. "Hepatic progenitor cells in human liver diseases". Seminars in Cell and Developmental Biology 13.6 (2002): 389-396.

89. TA Roskams., et al. "Nomenclature of the finer branches of the biliary tree: canals, ductules, and ductular reactions in human livers". Hepatology 39.6 (2004): 1739-1745.

90. N D Theise., et al. "The canals of Hering and hepatic stem cells in humans". Hepatology 30.6 (1999): 1425-1433.

91. M Fujita., et al. "Sequential observation of liver cell regeneration after massive hepatic necrosis in auxiliary partial orthotopic liver transplantation". Modern Pathology 13.2 (2000): 152-157.

92. L E Schmidt and K Dalhoff. "Alpha-fetoprotein is a predictor of outcome in acetaminophen-induced liver injury". Hepatology 41.1 (2005): 26-31.

93. Michalopoulos GK. "Liver Regeneration". Journal of Cell Physiology 213.2 (2007): 286-300.

94. W Duncan., et al. "Stem cells and liver regeneration". Gastroenterology 137.2 (2009): 466-481.
95. M Huch., et al. "In vitro expansion of single Lgr5+ liver stem cells induced by Wnt-driven regeneration". Nature 494.7436 (2013): 247-250.

96. M Huch., et al. "Long-term culture of genome-stable bipotent stem cells fromadult human liver". Cell 160.1-2 (2015): 299312.

97. E W Kuijk., et al. "Generation and characterization of rat liver stem cell lines and their engraftment in a rat model of liver failure". Scientific Reports 6 (2016).

\section{Assets from publication with us}

- Prompt Acknowledgement after receiving the article

- Thorough Double blinded peer review

- Rapid Publication

- Issue of Publication Certificate

- High visibility of your Published work

Website: https://www.actascientific.com/

Submit Article: https://www.actascientific.com/submission.php

Email us: editor@actascientific.com

Contact us: +919182824667 